\title{
Modeling deuterium chemistry of interstellar space with large chemical networks
}

\author{
T. Albertsson ${ }^{1}$, D. A. Semenov ${ }^{1}$, A. I. Vasyunin ${ }^{2}$, Th. Henning ${ }^{1}$ and \\ E. Herbst ${ }^{2}$ \\ ${ }^{1}$ Max-Planck-Institut für Astronomie, Königstuhl 17, 69117 Heidelberg, Germany \\ email: albertsson@mia.de \\ ${ }^{2}$ Department of Chemistry, University of Virginia, Charlottesville, VA 22904, USA
}

\begin{abstract}
Observations of deuterated species are essential to probing the properties and thermal history of various astrophysical environments, and the ALMA observing facilities will reveal a multitude of new deuterated molecules. To analyze these new vast data we have constructed a new up-to-date network with the largest collection of deuterium chemistry reactions to date. We assess the reliability of the network and probe the role of physical parameters and initial abundances on the chemical evolution of deuterated species. Finally, we perform a sensitivity study to assess the uncertainties in the estimated abundances and $\mathrm{D} / \mathrm{H}$ ratios.
\end{abstract}

Keywords. astrochemistry, molecular processes, methods: numerical, ISM: clouds, ISM: molecules, stars: circumstellar matter, protostars

The life cycle of molecules covers a wide range of environments, starting from the sparse interstellar medium (ISM) that eventually evolves into stars and planets, and interstellar chemistry is the key to probing these environments. As molecular hydrogen cannot be observed in the cold ISM, other molecular tracers are employed to probe the relevant physical conditions and chemical composition. In this regard the study of deuterium chemistry has proven useful in constraining the ionization fraction, density and thermal history of the ISM and protoplanetary disks.

In the light of the high-sensitivity observations we can expect from ALMA, we present a up-to-date, extended, multi-deuterated chemical network consisting of $55000+$ reactions. We assess its reliability by the modeling of the deuterium fractionation in various phases of the ISM and comparing it with observed $\mathrm{D} / \mathrm{H}$ ratios of a variety of mono-, doubly-, and triply-deuterated species in distinct astrophysical environments. A list of the most promising, potentially detectable deuterated species with ALMA is also provided.

We find a good agreement with previous model studies, such as those by Roberts \& Millar (2000a) and Roberts \& Millar (2000b). Our model successfully explains the observed $\mathrm{D} / \mathrm{H}$ ratios for many molecules in the ISM, including water, methanol, ammonia and many hydrocarbons, and offers an improvement in reproducing the observations of $\mathrm{DCO}^{+}, \mathrm{HDCO}$, and $\mathrm{D}_{2} \mathrm{CO}$ as compared to previous models.

Lastly, we studied uncertainties in calculated abundances, and hence column densities, as well as $\mathrm{D} / \mathrm{H}$ ratios for deuterated species. Abundance uncertainties for up to triplydeuterated species with relative abundances $>10^{-25}$ are shown in the left plot of Figure 1, and the same for $\mathrm{D} / \mathrm{H}$ ratios is shown in the right plot. We find that, on a $1 \sigma$ confidence level, the typical uncertainties for the calculated abundances, and hence column densities, are a factor of $1.25-5,3-10$ and approximately 10 for species made of $\lesssim 3,4-8$ atoms and $>8$ atoms, respectively. We find also that uncertainties of deuterated species are in general a factor of $\sim 2-3$ larger than those of their un-deuterated analogues. A lot of chemically simple species with high abundance uncertainties contain either $\mathrm{Mg}$, $\mathrm{Na}$, or 

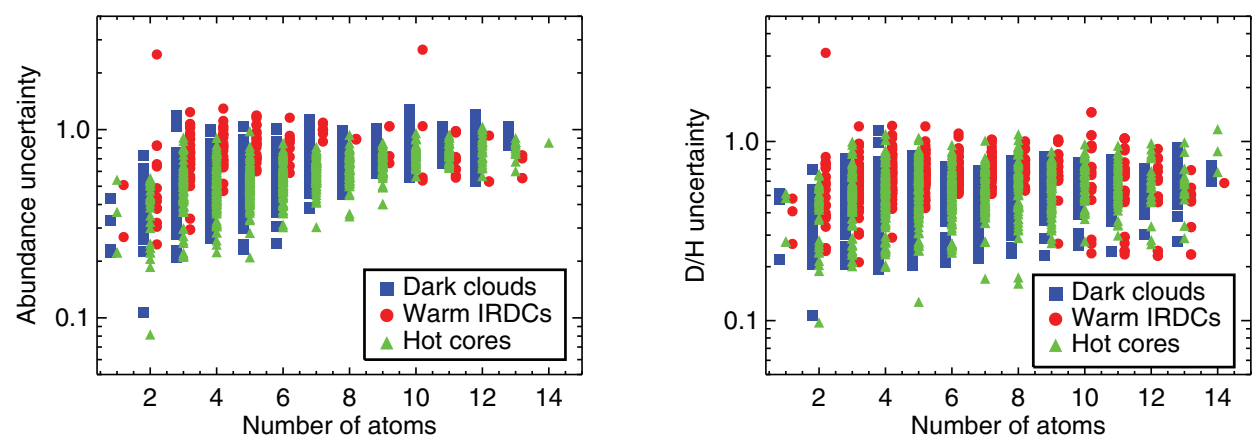

Figure 1. The $1 \sigma$ uncertainties of abundances (left) and $\mathrm{D} / \mathrm{H}$ ratios (right) for up to triply-deuterated species with relative abundances $>10^{-25}$ as a function of molecule size. Dark clouds are shown with (blue) squares, warm infrared clouds with (red) circles and hot cores with (green) triangles.

$\mathrm{Si}$, since the respective chemical pathways remain poorly investigated. We also find that large hydrocarbons $\left(\mathrm{C}_{n} \mathrm{H}_{m}, \mathrm{n} \gtrsim 4\right)$, despite being abundant (with relative abundances up to $\left.10^{-9}-10^{-7}\right)$, have large error bars in the computed abundances.

Overall the uncertainties in $\mathrm{D} / \mathrm{H}$ ratios are lower compared to that of the abundances of the corresponding $\mathrm{H}$ - and D-bearing isotopologues. This is because for a majority of deuterated reactions there are no measured rate coefficients, and instead estimates has to be made based upon their un-deuterated analogues. Hence, often abundances of the $\mathrm{H}$ - and D-bearing isotopologues both are similarly affected by the rate, which is approximately half an order of magnitude, and vary between a factor of 2 and 10. As in the case of the abundance uncertainties, we find the largest $\mathrm{D} / \mathrm{H}$ uncertainties for large hydrocarbons $\left(\mathrm{C}_{n} \mathrm{H}_{m}, \mathrm{n} \gtrsim 4\right)$ and species containing $\mathrm{Mg}, \mathrm{Na}$, and $\mathrm{Si}$.

We isolate the most problematic reactions for the isotopologues and isomers of $\mathrm{H}_{3}^{+}$, $\mathrm{HCO}^{+}, \mathrm{HCN}, \mathrm{H}_{2} \mathrm{O}, \mathrm{CH}_{3} \mathrm{OH}, \mathrm{H}_{3} \mathrm{O}^{+}, \mathrm{CH}_{3}^{+}, \mathrm{C}_{2} \mathrm{H}_{2}^{+}$and $\mathrm{CO}$, and find that this reaction list is dominated by ion-neutral and dissociative recombination reactions, most of them connected to the chemical evolution of water and methanol. Other essential reactions include the cosmic ray ionization of $\mathrm{H}_{2}$ and $\mathrm{He}$ and a small number of neutral-neutral reactions.

\section{Acknowledgements}

T. A. acknowledges the support of the European Community's Seventh Framework Programme [FP7/2007-2013] under grant agreement no. 238258. D.S. acknowledges support by the Deutsche Forschungsgemeinschaft through SPP 1385: "The first ten million years of the solar system - a planetary materials approach" (SE 1962/1-1 and SE 1962/1-2). E. H. acknowledges the support of the National Science Foundation (US) for his astrochemistry program, and the support of NASA for studies in the evolution of pre-planetary matter.

\section{References}

Roberts, H. \& Millar, T. J. 2000, A\&A 361, 388

Roberts, H. \& Millar, T. J. 2000, A\&A 364, 780 